\title{
Germanica
}

\section{Le modernisme dans la littérature enfantine en Suède. Quelques lignes directrices}

Der Modernismus in der Schwedischen Kinderliteratur. Einige charakteristische Züge

Lena Kåreland

Traducteur : Anna Bellinder-Broca

\section{OpenEdition}

Journals

Édition électronique

URL : http://journals.openedition.org/germanica/1495

DOI : $10.4000 /$ germanica. 1495

ISSN : 2107-0784

Éditeur

Université de Lille

Édition imprimée

Date de publication : 1 juin 1993

Pagination : 129-141

ISSN : 0984-2632

\section{Référence électronique}

Lena Kåreland, « Le modernisme dans la littérature enfantine en Suède. Quelques lignes directrices », Germanica [En ligne], 12 | 1993, mis en ligne le 21 mars 2014, consulté le 06 octobre 2020. URL : http:// journals.openedition.org/germanica/1495; DOI : https://doi.org/10.4000/germanica.1495

Ce document a été généré automatiquement le 6 octobre 2020.

(c) Tous droits réservés 


\section{Le modernisme dans la littérature enfantine en Suède. Quelques lignes directrices}

Der Modernismus in der Schwedischen Kinderliteratur. Einige charakteristische Züge

Lena Kåreland

Traduction : Anna Bellinder-Broca

\section{NOTE DE L'ÉDITEUR}

N.B. : Les titres en italique, cités en français entre parenthèses, ont été publiés en traduction française, les titres cités entre guillemets n'ont pas été traduits en français.

1 Comme point de départ de notre étude, nous prendrons l'œuvre de Marshall Berman sur le modernisme et la modernité, parue aux États-Unis en 1982 sous le titre All That is Solid Melts into Air. Berman y écrit notamment :

Être moderne, c'est de se trouver dans un environnement qui nous promet aventure, pouvoir, joie, croissance, transformation de nous-mêmes et du monde, et qui, parallèlement, nous menace de détruire tout ce que nous avons, tout ce que nous connaissons, tout ce que nous sommes ${ }^{1}$.

Berman résume ici le paradoxe inhérent au modernisme : d'une part la vie qui aspire à devenir art, et de l'autre l'homme qui cherche sa propre réalisation. Si on applique cette façon de voir à la littérature enfantine, elle fait ressortir la richesse et la multiplicité du genre. Même sa parenté avec la littérature pour adultes apparaît dans une autre lumière.

Concevoir l'art et la littérature comme quelque chose qui concerne par-dessus tout la vie de l'individu, nous permet de toucher au cœur même de la littérature enfantine. Autrement que le livre pour adultes, le livre pour enfants a toujours eu, et a encore 
aujourd'hui, un but pédagogique dans le sens large du mot. Il doit agir sur la vie de l'enfant, l'aider à prendre ses repères, développer son esprit, son imagination, le rendre plus heureux, bref, l'aider à vivre et à grandir.

4 Un des signes distinctifs du modernisme est la focalisation sur l'individu et le culte du Moi. Fifi Brindacier, créée par Astrid Lindgren, est indiscutablement le premier personnage véritable-ment moderne de la littérature enfantine suédoise, Fifi s'efforce constamment de rester libre, par rapport aux parents aussi bien que vis-à-vis des conventions. Elle incarne le Moi sans entraves, sans masques, sans hypocrisie, libre des déformations sociales. Fifi personnifie le chaos fertile. Elle symbolise la vie authentique, le vrai et le créatif. L'aspect provocateur du personnage reste vivace, même à l'heure du postmodernisme, comme le souligne un spécialiste suédois de la littérature enfantine ${ }^{2}$.

5 Le modernisme en tant que courant littéraire peut être défini de différentes façons. Le plus souvent il résume un ensemble de tendances ou -ismes apparus au cours du $\mathrm{XX}^{\mathrm{e}}$ siècle. La naissance en Suède du livre moderne pour enfants est généralement datée de la fin de la guerre, en 1945, l'année où parut notamment Pippi Längstrump (Fifi Brindacier). La nouvelle orientation se carac-térise entre autres par le fait que les auteurs se solidarisent avec l'enfant, prennent son parti contre l'autorité des adultes. Le monde est vu et décrit dans la perspective de l'enfant. La révolte, le défi, même l'anarchie trouvent pour la première fois leur place dans le livre pour enfants.

Le modernisme dans la littérature suédoise pour adultes est apparu plus tôt, environ vers 1910. Il s'agit alors principalement de poésie et le terme se rapporte surtout à ses aspects formels. Des critères courants en sont le rythme libre et un langage imagé et condensé. Parmi les pionniers du modernisme suédois, on cite généralement Pär Lagerkvist ${ }^{3}$. La conception suédoise se distingue toutefois considérablement de celle qui a cours dans le débat international sur le sujet, où l'on ne tient pas uniquement compte de l'aspect formel mais aussi du contenu. Le modernisme n'est pas seulement une question d'esthétique mais comme nous l'avons vu, engage aussi une conception de l'homme et de la société4.

\section{Une nouvelle vision du monde}

7 Les exemples donnés dans cet aperçu de la littérature enfantine moderniste retiennent surtout l'aspect anticonformiste, le choix en faveur de la vie et de l'art et la remise en question de la tradition. En étudiant l'enfant et la relation enfant-parents telle qu'elle est décrite dans la littérature enfantine, on discerne une tendance intéressante. Cela est particulièrement vrai s'agissant du livre d'images ${ }^{5}$. Derrière l'image transformée de l'enfant se cache une nouvelle vision du monde et une nouvelle pédagogie que l'on pourrait définir comme la perte des points de repères et la déconstruction consciente. Le connu est dissous et cède la place à l'ambigu et à l'énigmatique. "La modernité est avant tout questionnement et (re)mise en question, interrogation permanente sur ellemême ", dit par exemple Alexis Nouss ${ }^{6}$.

8 Ces tendances se succèdent dans la littérature enfantine depuis la fin de la guerre. La dissolution des normes et des règles devient particulièrement évidente en ce qui concerne l'image de la famille. Le rôle parental est critiqué et mis en question. Dans la "nouvelle famille», la figure de l'ami se substitue à l'autorité des parents. Une « démocratisation » apparaît et l'instance parentale disparaît peu à peu. Dans certains 
cas, on pourrait même parler d'une exaltation de l'enfant dans le sillage de laquelle se profile une nouvelle vision romantique. L'enfant est vu comme le plus fort et dispose de ressources intérieures considérables. Comparé aux adultes, il est intègre, plus vivant et plus imaginatif. Il est capable de défendre son individualité, sa fraîcheur face à un entourage souvent coupable d'incompréhension et en dépit de la dérobade des parents. La description de l'enfant qui, malgré un départ défavorable dans la vie, ne se laisse pas "détruire ", devient fréquente. C'est l'apparition dans le livre pour enfants des «maskrosbarn» (littéralement «les enfants pissenlits»), des enfants aux taches de rousseur.

\section{La vitalité de l'enfant}

Cette attitude vis-à-vis de l'enfant, qui plonge ses racines dans le romantisme et les idées de J.-J. Rousseau, est manifeste par exemple dans le livre Det märkliga barnet (L'étrange enfant) de Ulf Nilsson, 1982. L'histoire, d'une tonalité sombre, qui conjugue poésie, imagination et un fort engagement social, est racontée par un petit garçon, le héros du livre. Le message contenu dans le récit est que la peur peut être surmontée, que l'on est en droit de s'élever contre l'autorité et de lui résister. La force morale se trouve chez l'enfant ${ }^{7}$.

La relation traditionnelle mère-enfant est fréquemment bouleversée dans les livres pour enfants modernes. Par sa force et sa vitalité, l'enfant apparaît comme celui qui domine, tandis que la mère paraît faible. C'est par exemple le cas dans les livres d'images qui racontent l'histoire du bébé terrible (Juju le bébé terrible) de Barbro Lindgren (texte) et Eva Eriksson (illustrations). À l'instar de Fifi Brindacier, le personnage du bébé est un héros moderne du livre pour enfants, d'une grande souveraineté et exprimant une grande soif de liberté. C'est un franc-tireur en coucheculotte qui met le cap sur la forêt, se laisse lécher par le loup sans peur aucune ${ }^{8}$. Il y a aussi chez le bébé des traits tyranniques et cruels. À beaucoup d'égards il opprime sa maman qui, à bout de fatigue, n'offre que peu de résistance. Cependant, la description de la mère est très nuancée et tranche avec celles des livres plus anciens, où la mère apparaît généralement comme l'autorité indiscutable. La maman du bébé terrible est représentée comme une femme indépendante, avec sa propre intégrité. Dans le rapport avec son enfant, elle n'est pas seulement la mère, mais aussi l'amie qui participe activement à ses jeux. Elle brime peu et s'implique beaucoup. Cette image de la mère toute en nuances, qui rompt avec les règles et les conventions, représente quelque chose de tout à fait nouveau dans la littérature enfantine suédoise.

11 Un réalisme acerbe, parfois à la limite de l'absurde, caractérise plusieurs albums suédois, par exemple Mammaboken («Le livre de la mère »), 1986, de Kristina Berge (texte) et Anna Höglund (illustrations). La mère, dans ce livre, offre une certaine ressemblance avec la maman du bébé terrible. L'une comme l'autre, malgré fatigue et lassitude, sont prêtes à accepter les conditions de l'enfant et à participer à ses jeux. Le livre est construit comme une suite de tableaux représentant des scènes de la vie quotidienne d'une mère seule avec ses deux enfants. Souvent il s'agit de situations conflictuelles où les enfants se mettent en scène et poussent leur mère à bout, ou bien, profitant de la situation, dressent les parents l'un contre l'autre.

12 L'observation de la réalité quotidienne glisse vers le surréalisme et comporte une dimension d'humour noir. Les illustrations soulignent ce côté grotesque et surréaliste. 
La gamme de couleurs est froide et les personnages dépeints de manière caricaturale. Un des enfants a, par exemple, une carotte en guise de nez. L'histoire se déroule dans un environnement aride, dénudé, sans détails. Les albums de Barbro Lindgren et Eva Eriksson ainsi que celui de Kristina Berge et Anna Höglund rompent avec les normes en vigueur. Ils véhiculent, à l'instar du modernisme, des forces révolutionnaires et chaotiques sous-jacentes?.

\section{Un monde renversé}

Le lieu central de l'histoire de Mammaboken est la cuisine de la famille. Le fait que, dans les deux situations qui s'y présentent, les enfants agissent seuls, en dit long sur la relation mère-enfant. La première fois, c'est le matin et l'un des enfants est sagement assis à la table de la cuisine devant la fenêtre sans rideaux. Il regarde son verre de lait renversé. L'autre enfant ouvre le réfrigérateur totalement vide. La scène est pathétique. La mère est absente mais le texte nous dit où elle se trouve :

Dans la chaleur du creux de son lit, petite Maman se plaît. Dans le monde des rêves, elle voudrait y rester.

Les enfants ont faim et voudraient manger, mais «maman dort comme si elle était morte ». La scène qui suit nous montre comment les enfants réveillent leur mère brutalement. Nous sommes introduits dans la chambre à coucher où la mère repose, des bigoudis dans les cheveux. La mère, dont un des enfants essaie ensuite de la nourrir avec du café et des toasts, ressemble davantage à une menace qu'à un acte d'amour. Pendant ce temps-là, l'autre enfant saute rageusement sur le ventre de la mère tout en jouant du rock'n'roll avec les couvercles de casserole. Par terre sont jetés des escarpins rouges, les talons cassés, et dans un coin traîne un tube d'aspirine. Est-ce parce que la mère est allée faire la fête la veille qu'elle n'arrive pas à se réveiller? L'auteur et surtout l'illustrateur montrent que la mère a droit à une vie privée à côté de son rôle de mère, un rôle que, parfois, elle fuit par révolte et par fatigue.

Ce livre nous montre également un monde où les rôles sont inversés. Parfois les enfants endossent le rôle d'adultes, tandis que la mère se comporte en enfant. C'est, par exemple, le cas de la scène finale, où les enfants se retrouvent de nouveau seuls. Ils se font face à la table de la cuisine, chacun est absorbé par la lecture d'un livre. Le calme et l'harmonie règnent. La mère, mise au lit par les soins de ses enfants, dort calmement dans la chambre voisine. Dehors, le ciel rougeoyant indique la fin de la journée et le début de la nuit. Le texte dit :

Les étoiles scintillent et la lune brille.

Dans notre cuisine personne n'a froid.

Nous lirons et parlerons aussi longtemps que nous voudrons.

Maman dort tranquillement dans la chambre à côté.

16 Le livre met en évidence la fragilité de l'existence et l'abandon qui est souvent le lot des enfants des années quatre-vingt. En même temps il insiste sur leur force et leur indépendance. Mammaboken, ainsi que le livre sur le bébé terrible, montre comment les frontières entre le monde des enfants et celui des adultes s'effacent aujourd'hui progressivement. Les adultes sont infantilisés tandis que les enfants se comportent en adultes de plus en plus tôt. Le temps de l'enfance se réduit peu à peu. Un autre exemple d'enfants volontaires et dynamiques, qui commandent à leurs parents, et non l'inverse, nous est donné dans Det underbara dagishemmet (« La crèche 
merveilleuse »), 1987, de Gun-Britt Sundström (texte) et Gunna Grähs (illustrations), où le caractère superflu du rôle des parents et de la cellule familiale traditionnelle est efficacement démontré. Nous suivons la petite Victoria, le personnage principal, depuis sa naissance. Le livre, plein d'humour, par moments franchement absurde, chante les louanges de la garde collective des enfants telle qu'elle est conçue dans les «daghem » suédois.

\section{Des mères version Fifi Brindacier} débridée. Il s'agit encore d'une mère seule et très peu conformiste, considérée comme le mouton noir dans sa famille. Déjà son aspect extérieur, ses grands cheveux ébouriffés, ses bas rayés, évoque Fifi Brindacier. Elle a l'air de s'être enfuie d'un cirque et rien que sa façon de marcher en dit long sur son attitude devant la vie. Elle ne marche pas comme les autres mamans, mais avance la plupart du temps en sautant, en bondissant. Quand elle fait de la pâtisserie c'est une sorte de fureur où la pâte vole, se colle au plafond ou tombe directement dans la bouche de celui qui se trouve être dans la cuisine.

La Studsmamma ressemble davantage à un enfant qu'à une personne adulte. Elle ne travaille pas comme les autres mamans et ses petits pains au sucre ne seront jamais mis au four. Ils seront mangés bien avant. Sa petite fille Lajjan a davantage un comportement d'adulte. À un moment la fille fait remarquer à sa mère que plutôt que des gâteaux, elle aimerait avoir des chaussettes neuves puisque les siennes sont trouées. Sa mère lui répond alors avec fermeté que, pour l'âme, les gâteaux, c'est beaucoup plus important que des chaussettes sans trous. 


\section{L'individualisme et réalité fragmentaire} qui ne prennent pas leurs responsabilités d'adultes. Cette critique est, la plupart du temps, masquée par l'humour ou l'ironie. Ainsi dans Nissejakten (« Le visage perdu de Nisse »), 1985, de Håkan Jaensson et Arne Norlin (texte) et Gunna Grähs (illustrations), l'existence des parents, rigide et planifiée au détail près, leur manque de temps pour leurs enfants, sont durement fustigés. Nissejakten, une histoire sur les chapeaux de roues, raconte le stress du matin dans la maison de Nisse. Chaque matin Nisse est expédié à toute vitesse chez sa grand'mère. Celle-ci, à la différence des parents, a du temps à consacrer à l'enfant. Un matin, la grand'mère remarque que quelque chose ne va pas : " Mon garçon, je crois bien que tu as perdu ta figure », déclare-t-elle. L'histoire $\mathrm{du}$ livre consiste alors à rechercher les différentes parties du visage du petit garçon. C'est une description symbolique du quotidien stressé de l'homme moderne, où il n'y a plus de place pour l'enfant. Nisse se sent tellement abandonné que même son identité s'efface. Il perd son visage. Mais, dans ce sentiment d'abandon, il y a aussi une force. Nisse est à la fois héros et anti-héros et sensiblement plus clairvoyant que ses parents. Le thème est abordé avec une ironie crue, en particulier dans les illustrations. L'égoïsme des parents est démasqué, et dans la scène finale, où on les voit la tête sous le bras, ils sont mis au pied du mur par la grand'mère et Nisse.

e côté puéril des parents, surtout celui de la mère, est particulièrement souligné dans les livres pour de très jeunes enfants. Ici le comportement non-adulte, ludique, est décrit comme quelque chose de positif. En revanche, dans les livres pour des enfants plus grands ou dans la littérature pour l'adolescence, le ton est plus critique. La relation enfants-parents devient plus complexe. «Pourquoi ne peux-tu pas être comme toutes les autres mamans! » s'exclame désespérément Simone, douze ans, dans Dàrfinkar och dönickar («Loufoques et mollassons »), 1984, de Ulf Stark. Cette comédie bouffonne, qui a été primée et filmée, décrit une mère bohème, désordonnée et distraite, en même temps que chaleureuse et très humaine. 
Ulf Stark fait partie des auteurs les plus remarqués dans le domaine de la littérature pour l'enfance et la jeunesse des années quatre-vingt, et l'on retrouve dans ses livres la plupart des tendances du genre pendant cette période. Un évident esprit citadin, qui mêle le réalisme et l'absurde dans la description de la vie familiale, marque ses livres. Dans Karlavagnen ("La grande Ourse »), 1989, la mère égocentrique et incapable de s'installer quelque part, ressemble à bien d'autres personnages de la littérature enfantine des années quatre-vingt. Il est significatif que son morceau de musique préféré soit born to run, en même temps épigraphe du livre. Le personnage principal Tias et sa petite sœur sont habitués à ce que leur mère déménage systématiquement au moment où eux-mêmes commencent à s'adapter et à s'installer.

Aussi longtemps qu'il se souvienne, ils ont toujours été en route pour quelque part. Et de cela il en a assez. A peine commence-t-il à se faire des copains que maman ne tient plus en place et qu'il faut encore faire des bagages. Elle est comme ça, tout simplement, une espèce de vagabond, persuadée qu'il suffit de partir pour que tout aille mieux ${ }^{13}$.

\section{Le parent absent}

Dans Tiga är guld («Le silence est d'or»), 1991, de Cannie Möller, nous faisons la connaissance de Lisa qui adopte un rôle maternel à l'égard d'un de ses parents. La mère est alcoolique et sa fille doit souvent faire fonction de parent ; éviter que la mère ne soit en retard, lui rappeler des choses, faire la cuisine ou mettre un peu d'ordre dans la maison. La mère pense d'abord à elle-même et à sa carrière d'actrice.

La trahison des adultes marque également la vie de Jonn telle qu'elle est décrite dans Jonns resa («Le voyage de Jonn »), 1988, et Jonns andra resa ( Le deuxième voyage de Jonn »), 1991, de Håkan Jaensson. Dans le premier livre, la mère de Jonn, qui est maniaco-dépressive, s'enfuit en abandonnant Jonn et son travail de caissière dans un grand magasin. Se sentant responsable de sa mère, Jonn part à sa recherche en faisant du stop du sud de la Suède jusqu'à Stockholm. Dans le deuxième livre la famille est de nouveau réunie et en vacances en caravane. Mais le bonheur familial n'est toujours pas au rendez-vous. Les parents ne s'entendent pas et se querellent. La mère ne supportant pas la vie dans la caravane, qu'elle vit comme une prison, s'enfuit de nouveau, sans but précis, mais cette fois-ci en emmenant son fils avec elle.

C'est le début d'un voyage à travers la Suède, une sorte de récit picaresque qui nous donne un aperçu de la vie quotidienne dans la Suède d'aujourd'hui, vue des autoroutes, des gares de chemin de fer et des petites villes de province. Jonn est désespéré et déçu par sa mère. Afin de maîtriser son désespoir, il sort de lui-même et s'efforce de voir de l'extérieur ce qui leur arrive à lui et à sa mère. Leur vie se déroule comme sur un écran de cinéma ou sur une scène de théâtre. Il observe les familles en vacances dans d'autres caravanes et rêve désespérément de faire partie d'une famille heureuse, ou du moins d'une famille normale, où l'on fait de la pâtisserie et où l'on possède un four à microondes. Avec intensité et un grand sens du détail, l'auteur nous livre des tranches de vie suédoises en même temps qu'il montre la fragilité et les difficultés de l'homme à maîtriser son existence.

Les personnages mentionnés ci-dessus, des enfants volontaires et forts et des enfants utopiques, représentants de la révolte et du changement, peuvent être considérés comme des symboles du héros moderne, œuvrant et agissant dans un environnement 
qui, selon la définition de Berman, promet "aventure, pouvoir, joie, croissance et transformation d'eux-mêmes et du monde ». Ils libèrent des forces chez eux-mêmes et dans leur entourage.

\section{Un héros à la Hamlet}

31 L'évolution du roman suédois pour la jeunesse, au cours des années quatre-vingt, va du réalisme social au réalisme fantastique avec des éléments de mysticisme et des descriptions psycho-logiques où l'accent est mis sur des questions d'ordre existentiel.

Une relation complexe entre mère et fils est au centre des livres pour la jeunesse de Mats Wahl comprenant Halva sanningen («La moitié de la vérité »), 1984, Jiggen, 1985 et Den lackerade apan ("Le singe laqué »), 1986. Le personnage principal de la trilogie est un adolescent de Stockholm, Harry Stockman. Ici c'est l'absence de contact entre parents et enfants qui domine, le thème du livre étant la haine envers la mère. C'est également un texte sur la solitude et l'isolement. Harry est un personnage compliqué, qui cherche désespérément un point d'ancrage dans l'existence. Son attitude, en apparence dure et violente, cache mal son désespoir et le chaos sentimental dont il est la victime. Il est capricieux, égocentrique et manifeste constamment une activité fébrile. Mais c'est aussi un flâneur et un marginal qui regarde passer la vie sans vraiment y participer.

Son rapport à sa mère est blessé et tendu. Il profite d'elle, notamment en ce qui concerne l'argent, lui ment constamment. Ils sont pris dans un rapport de méfiance mutuelle, incapables de communiquer et de se comprendre. La mère, sans imagination, imbue de préjugés, apparait comme un personnage profondément tragique dans sa solitude et sa tristesse. Harry, de son côté, ne croit en rien et n'a ni valeurs ni ligne de conduite. Son désarroi est celui d'un héros moderne, instable et engagé dans une quête permanente.

34 L'instance parentale est en voie de disparition et la communauté familiale une illusion, c'est la leçon qui se dégage des livres de Mats Wahl. Les mécanismes psychologiques qui sous-tendent les liens sentimentaux entre les membres d'une famille et les tendances destructives qui peuvent s'y développer sont habilement mis à $n u^{14}$. Il importe néanmoins de souligner une différence importante entre la littérature pour jeunes enfants et celle pour adolescents. Dans le premier cas nous sommes en général en présence d'un enfant fort et indomptable, qui suit son propre chemin, alors que le héros du livre pour adolescents se présente davantage comme une sorte de Hamlet, d'une nature éclatée, indécise, apathique, sans foi en l'avenir. Il remet tout en question, continuellement à la recherche de quelque chose ou en route vers un ailleurs. À l'instar du petit enfant il se trouve néanmoins dans un processus dynamique qui développe et accomplit le Moi. Dans ce sens la littérature enfantine en Suède correspond bien à l'idéologie du modernisme, qui se caractérise entre autres par une continuelle évolution ${ }^{15}$. 


\section{NOTES}

1. Citation tirée de la traduction suédoise. Berman, Marshall, «allt som är fast förflyktigas », Modernism och modernitet, Stockholm : Arkiv förlag, 1987, p. 13.

2. Edström, Vi., «Pippi Längstrump - kaos och postmodernism», dans: Poesi och vetande, Stockholm : Norstedt, 1990, p. 229.

3. Den svenska Utteraturen. Modernister och arbetare, tome V, rédigé par Lars Lönnroth et Sven Delblanc, Stockholm : Bonniers, 1989, p. 97.

4. Luthersson, Peter, « När modernismen inte kom till Sverige », dans : Allt om böcker, 1991, 3, pp. 4-11.

Dans cet artide, intéressant dans son principe, Luthersson livre une reflexion sur la definition du modernisme dans l'histoire suédoise qui, selon lui, se fond trop exclusivement sur l'exemple de Lagerkvist si on la compare à celle généralement admise sur le plan international.

5. Cf. Ulla Rhedin dans Vår moderna bilderbok, Stockholm : Rabén \& Sjögren, 1991, pp. 155-187. Le livre a été édité à l'occasion de l'exposition célébrant 400 ans de littérature enfantine en Suède, organisée par le Musée national de Stockholm en 1991.

6. Nouss, Alexis, La modernité, Paris : Jacques Grancher, 1991, p. 12.

7. Kåreland, Lena, "Swedish Children's Books in the eighties. Tradition and change ", dans : Scandinavian Public Library Quarterly, 1990, 1, vol. 23, p. 25.

8. Bäckström, Lars, Mannen utan väg och hans Kusin Vitamin. Om barn- och ungdomslitteratur; ibland under jämförelse med de vuxnas litteratur, Stockholm : Rabén \& Sjögren, 1991, p. 22.

9. Nouss, Alexis, La modernité, 1991, p. 29.

10. Ibidem, p. 51.

11. Ibidem, p. 95.

12. Hassan, Ihab, « Mot ett begrepp om postmodernism», dans : Postmoderna tider ? Red. Mikael Löfgren \& Anders Molander, Stockholm : Norstedt, 1986, p. 74.

13. Stark, Ulf, Karlavagnen, Stockholm : Bonniers, 1989, p. 8.

14. Kåreland, Lena, «Swedish Children's Books in the eighties. Tradition and change », 1990, p. 29.

15. Nouss, Alexis, La modernité, 1991, p. 95.

\section{RÉSUMÉS}

Un des signes distinctifs les plus marqués du modernisme est la focalisation sur l'individu et le culte du Moi. Cela caractérise aussi la littérature enfantine suédoise. Fifi Brindacier, créée par Astrid Lindgren, est sans doute le premier personnage véritablement moderne de cette littérature en ce sens qu'elle s'efforce constamment de rester libre, par rapport aux parents aussi bien que vis-à-vis des conventions.

Les exemples donnés dans cet aperçu de la littérature enfantine moderniste retiennent surtout l'aspect anticonformiste. En étudiant l'enfant et la relation enfant-parents, on discerne une nouvelle vision du monde et une nouvelle pédagogie que l'on pourrait définir comme la perte des points de repères et la déconstruction consciente. Le rôle parental est critiqué et mis en question. La figure de l'ami se substitue à l'autorité des parents. En même temps une démocratisation 
apparaît et on pourrait même parler d'une exaltation de l'enfant. Une nouvelle vision romantique se profile où l'enfant est le plus vivant et le plus fort.

Dans la littérature enfantine moderniste en Suède, on trouve également un type de mère que l'on pourrait appeler « la mère à la Fifi Brindacier ». Contrairement au rôle traditionnel de la mère, celle-ci incarne la disponibilité et l'ouverture au changement propres au modernisme. Le côté puéril des parents, surtout celui de la mère, est particulièrement souligné dans les livres pour de très jeunes enfants. En revanche, dans la littérature pour l'adolescence le ton est plus critique. Le héros, ici, se présente comme une sorte de Hamlet, d'une nature éclatée, indécise et sans foi en l'avenir. Cela étant, l'enfant comme l'adolescent sont pris dans un processus dynamique qui développe et accomplit leur Moi, trait caractéristique du modernisme.

Eines der Kennzeichen des Modernismus sowie der modernen schwedischen Kinder literatur ist die Betonung des Individuums und der Kult des Ichs. Pippi Langstrumpf von Astrid Lindgren ist zweifellos der erste moderne Held in Kindersocken der sich bemüht frei zu bleiben, nicht nur von der Eltern, sondern auch von allen Konventionen.

Die Beispiele der modernistischen Kinderliteratur, die in dieser Übersicht gegeben sind, beschäftigen sich besonders mit dem Antikonformismus und dem Antitraditionalismus. Wenn man in den Kinderbüchern die Beziehungen zwischen Kindern und Eltern studiert, kann man eine neue Auffassung des Kindes und der Kindheit, wie auch der Kinderliteratur entdecken. Diese Auffassung kann man als eine bewußte Desorientierung und Dekonstruktion beschreiben. Die Rolle der Eltern wird kritisiert und diskutiert. Sie ist nicht mehr so autoritär wie früher, sondern ist freundlicher geworden. Eine romantische Auffassung des Kindes erscheint, man betrachtet das Kind als lebendiger und kraftvoller als den Erwachsenen.

In der modernen schwedischen Kinderliteratur findet man auch viele Beispiele der sogenannten « Mütter à la Pippi Langstrumpf ». Diese Mütter beweisen eine Bereitwilligkeit ihre Rolle neu zu verstehen, die charakteristisch für den Modernismus ist. Die naive Seite der Eltern, besonders die der Mutter, tritt vor allem zutage in Büchern für die kleinen Kinder. In der Jugendliteratur dagegen werden die Eltern oft kritisiert. Der Held der Jugendliteratur wird wie ein Hamlet dargestellt, unentschlossen und ohne Glauben an die Zukunft. Das Kind sowie der junge Mensch befinden sich doch in einem dynamischen Prozess, der die Persönlichkeit entwickelt und der den Spielraum für das Ich erweitert. 